\title{
Orbital entanglement and violation of Bell inequalities in mesoscopic conductors
}

\author{
P. Samuelsson, E.V. Sukhorukov and M. Büttiker \\ Département de Physique Théorique, Université de Genève, CH-1211 Genève 4, Switzerland.
}

\begin{abstract}
We propose a spin-independent scheme to generate and detect two-particle entanglement in a mesoscopic normal-superconductor system. A superconductor, weakly coupled to the normal conductor, generates an orbitally entangled state by injecting pairs of electrons into different leads of the normal conductor. The entanglement is detected via violation of a Bell inequality, formulated in terms of zero-frequency current cross-correlators. It is shown that the Bell inequality can be violated for arbitrary strong dephasing in the normal conductor.

PACS numbers: 74.50.+r, 73.50.Td, 73.23.Ad
\end{abstract}

Entanglement is one of the most intriguing features predicted by quantum theory [1]. It leads to correlation between distant particles, which can not be described by any local, realistic theory [2]. This nonlocal property of entanglement has been demonstrated convincingly in optics [3], where entangled pairs of photons have been studied over several decades. Apart from the fundamental aspects, there is a growing interest in using the properties of entangled particles for applied purposes, such as quantum cryptography 4 and quantum computation [5].

Recently, much interest has been shown for entanglement of electrons in solid state devices. A controlled generation and manipulation of electronic entanglement is of importance for a large scale implementation of quantum information and computation schemes. Electrons are however, in contrast to photons, massive and electrically charged particles, which raises new fundamental questions and new experimental challenges. Existing suggestions are based on creating 6, 7], manipulating and detecting [8, 9, 10] spin-entangled pairs of electrons. This requires experimental control of individual spins via spin filters or locally directed magnetic fields on a mesoscopic scale. Here we propose a spin-independent scheme for creating and detecting orbital entanglement in a mesoscopic normal-superconductor system.

We show that a superconductor, weakly coupled to a normal conductor (see Fig. 1), creates an orbitally entangled state by emitting a coherent superposition of pairs of electrons into different leads of the normal conductor. The zero-frequency correlation between currents flowing into different normal reservoirs is shown to be equivalent to a pair coincidence measurement: only correlations between the electrons from the same entangled pair contribute. As a consequence, a standard Bell Inequality (BI) can be directly formulated in terms of the zero-frequency current correlators. We find that a violation of the BI, demonstrating the entanglement of the pair state, can be obtained for arbitrary dephasing in the normal conductor.

We first consider a simplified version of the system (see Fig. 1), a more detailed discussion is given below.
A single 11 superconductor (S) is weakly coupled to a normal conductor, a ballistic two-dimensional electron gas, via two tunnel barriers 1 and 2 with transparency $\Gamma \ll 1$. The normal conductor consists of four arms, $1 A, 1 B, 2 A$ and $2 B$, with equal lengths $L$. The arms $1 A$ and $2 A(1 B$ and $2 B)$ are crossed in a controllable beam splitter $A(B)$, parameterized via the angle $\phi_{A}\left(\phi_{B}\right)$, and then connected to normal reservoirs $+A$ and $-A(+B$ and $-B)$. The beam splitters $[12$ are assumed to support

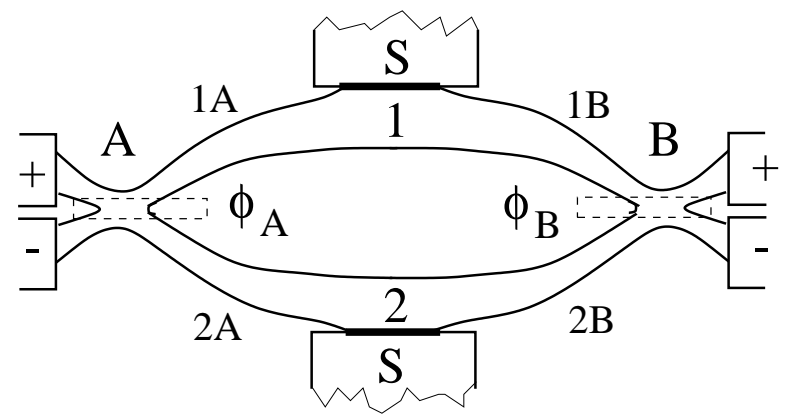

FIG. 1: The system: A single superconductor (S) is connected to four normal arms via two tunnel barriers 1 and 2 (thick black lines). The arms are joined pairwise in beam splitters $\mathrm{A}$ and $\mathrm{B}$ and end in normal reservoirs + and - .

only one propagating mode. The states $|+, \eta\rangle$ and $|-, \eta\rangle$ for electrons going out into the normal reservoirs and the states $|1, \eta\rangle$ and $|2, \eta\rangle$ of the electrons emitted from superconductor are related via a scattering matrix:

$\left[\begin{array}{l}|+, \eta\rangle \\ |-, \eta\rangle\end{array}\right]=\left(\begin{array}{cc}\cos \phi_{\eta} & -\sin \phi_{\eta} \\ \sin \phi_{\eta} & \cos \phi_{\eta}\end{array}\right)\left[\begin{array}{l}|2, \eta\rangle \\ |1, \eta\rangle\end{array}\right], \eta=A, B .(1)$

The angles $\phi_{A}$ and $\phi_{B}$ can be tuned between 0 and $\pi / 2$ by tuning the beam splitter from "open", when the electrons are transmitted through from 1(2) to $-(+)$, to "closed", when the electrons are completely reflected, from $1(2)$ to $+(-)$. We consider the low temperature limit, $k T \ll e V$. A negative voltage $-e V$ is applied to all the normal reservoirs and the superconductor is grounded. The voltage $\mathrm{eV}$ is smaller than the superconducting gap $\Delta$, so no single particle transport takes place. It is further assumed that the size of the system is smaller than the phase breaking length. 
We first present a simple and transparent explanation of how the entanglement is generated and detected, a rigorous derivation follows below. The superconductor emits pairs of particles into the normal arms. Since the superconductor is a single object, coherent on a macroscopic scale, the state of an emitted pair is a linear superposition of states corresponding to a pair emitted through barrier 1 and 2. The emitted pair can either split, with one electron going to each beam splitter $A$ and $B$, or both electrons can go to the same beam splitter. However, the latter process does not contribute 13] to the quantity of interest, the current cross-correlations, to leading order in pair emission (Andreev reflection) probability, proportional to $\Gamma^{2}$. The relevant part of the state of the emitted pair can thus be written as

$$
|\Psi\rangle=\left|\Psi_{12}\right\rangle \otimes\left|\Psi_{A B}\right\rangle, \quad\left|\Psi_{12}\right\rangle=(|11\rangle+|22\rangle) / \sqrt{2},
$$

a product of a state $\left|\Psi_{12}\right\rangle$, orbitally entangled with respect to emission across barrier 1 and 2 (called 12-space below), and a state $\left|\Psi_{A B}\right\rangle$, describing one electron going towards $A$ and one towards $B$, containing all additional information, such as energy and spin dependence. The beam splitters $A$ and $B$, parameterized via the angles $\phi_{A}$ and $\phi_{B}$, rotate the state $\left|\Psi_{12}\right\rangle$ [see Eq. (10].

The entanglement is detected by violation of a BI. We recall that in the original formulation [2], a source emitting spin- $1 / 2$ singlets was considered. The BI, as formulated in Ref. [14],

$$
\begin{aligned}
S & \equiv \mid E\left(\phi_{A}, \phi_{B}\right)-E\left(\phi_{A}, \phi_{B}^{\prime}\right) \\
& +E\left(\phi_{A}^{\prime}, \phi_{B}\right)+E\left(\phi_{A}^{\prime}, \phi_{B}^{\prime}\right) \mid \leq 2
\end{aligned}
$$

is expressed in terms of spin correlation functions [15]

$$
E\left(\phi_{A}, \phi_{B}\right)=P_{++}-P_{+-}-P_{-+}+P_{--} .
$$

Here $P_{\alpha \beta}\left(\phi_{A}, \phi_{B}\right)$ are the joint probabilities to observe one particle in detector $A$ with a spin $\alpha= \pm[+(-)$ denoting up (down)] along the $\phi_{A}$-direction, and the other in detector $B$ with a spin $\beta= \pm$ along the $\phi_{B}$-direction. The joint probabilities are given by

$$
P_{\alpha \beta}\left(\phi_{A}, \phi_{B}\right)=1+\alpha \beta \cos \left[2\left(\phi_{A}-\phi_{B}\right)\right] .
$$

Inserting the values for $P_{\alpha \beta}$ from Eq. (5) into Eq. (4), we get $E\left(\phi_{A}, \phi_{B}\right)=\cos \left[2\left(\phi_{A}-\phi_{B}\right)\right]$. We then directly see that for e.g. angles $\phi_{A}=\pi / 8, \phi_{B}=\pi / 4, \phi_{A}^{\prime}=3 \pi / 8$ and $\phi_{B}^{\prime}=\pi / 2$, the BI in Eq. (3) is maximally violated, i.e. we get $S=2 \sqrt{2}$.

In our orbital setup (see Fig 1), it is clear from Eq. (2) that the 12-space plays the role of a pseudo-spin space and the normal reservoirs act as detectors. We can thus, in direct analog to Ref. [2], formulate a BI in terms of any observable which is directly proportional to the corresponding joint probability $P_{\alpha \beta}\left(\phi_{A}, \phi_{B}\right)$ for our state $\left|\Psi_{12}\right\rangle$ [here $\alpha, \beta= \pm$ denote the reservoirs, see Fig.
1. We find below that the zero-frequency current crosscorrelator is given by

$$
S_{\alpha \beta} \equiv 2 \int_{-\infty}^{\infty} d t\left\langle\delta \hat{I}_{\alpha A}(t) \delta \hat{I}_{\beta B}(0)\right\rangle=P_{0} P_{\alpha \beta}\left(\phi_{A}, \phi_{B}\right)
$$

i.e. directly proportional to the joint probability distribution. Here $P_{0}=e^{3} V \Gamma^{2} / 2 h$, and $\delta \hat{I}_{\alpha \eta}(t)=\hat{I}_{\alpha \eta}(t)-\left\langle\hat{I}_{\alpha \eta}\right\rangle$ is the fluctuating part of the current $\hat{I}_{\alpha \eta}(t)$ in reservoir $\alpha \eta$. This leads to the important result that the Bell inequality in Eq. (3) can be directly formulated in terms of the zero-frequency current correlators in Eq. (6). We note [15] that when inserting $S_{\alpha \beta}$ directly into the correlation functions Eq. (4) (i.e. substituting $P_{\alpha \beta}$ with $S_{\alpha \beta}$ ), we must divide by the sum of all correlators $S_{++}+S_{+-}+S_{-+}+S_{--}=P_{0}$, which just eliminates $P_{0}$.

The simple result in Eq. (6) can be understood by considering the properties of the time-dependent correlator $\left\langle\delta \hat{I}_{\alpha A}(t) \delta \hat{I}_{\beta B}(0)\right\rangle$. It is finite only for times $t \lesssim \tau_{c}$, where $\tau_{c}=\hbar / \mathrm{eV}$ is the correlation time of the emitted pair (see Fig. 2). In the tunneling limit under consideration, $\Gamma \ll 1$, the correlation time is much smaller than the average time between the arrival of two pairs $e / I \sim \hbar / e V \Gamma^{2}$. As a result, only the two electrons within a pair are correlated with each other, while electrons in different pairs are completely uncorrelated. Thus, the zero frequency current correlator in Eq. (6) is just a coincidence counting measurement running over a long time, collecting statistics over a large number of pairs [16].

For a rigorous derivation of the above result, we first discuss the role of the superconductor as an emitter of pairs of orbitally entangled electrons. Since the system is phase coherent, we can work within the scattering approach to normal-superconducting systems [17, 18]. The starting point is the many-body state of the normal reservoirs, describing injection of hole quasiparticles at energies from 0 to $\mathrm{eV}$ (suppressing spin notation),

$$
\left|\Psi_{i n}\right\rangle=\prod_{0<E<e V} \gamma_{1 A}^{\dagger}(E) \gamma_{2 A}^{\dagger}(E) \gamma_{1 B}^{\dagger}(E) \gamma_{2 B}^{\dagger}(E)|0\rangle,
$$

where the ground state $|0\rangle$ is the vacuum for quasiparticles in the normal reservoir. The operator $\gamma_{1 A}^{\dagger}(E)$ creates a hole plane wave at energy $E$ (counted from the superconducting chemical potential $\mu_{S}$ ) in lead $1 A$, going out from the normal reservoirs towards the superconductor, and similarly for the other operators. The commutation relations for the $\gamma$-operators are standard fermionic.

To obtain the state of the quasiparticles going out from the superconductor, $\left|\Psi_{\text {out }}\right\rangle$, we note that the operators creating and destroying quasiparticles going out from the superconductor (towards the normal reservoirs) are related [17, 19] to the operators of the incoming quasiparticles via a scattering matrix. The scattering amplitude for a hole injected in arm $1 A$, to be back-reflected as an electron in arm $2 A$, is denoted $r_{2 A, 1 A}^{e h}$ and similarly for 
the other scattering amplitudes. In the tunnel limit under consideration, the amplitude to backscatter as the same type of quasiparticle is $r_{j \eta, j^{\prime} \eta^{\prime}}^{h h} \sim 1$. The amplitude for Andreev reflection, $r_{j \eta, j^{\prime} \eta^{\prime}}^{e h}$, is given by $-i \Gamma / 4$ (independent on energy). Processes where a hole incident on barrier 1(2) is backscattered as an electron at barrier $2(1)$, i.e. when a pair in the superconductor breaks up, are exponentially suppressed with the distance between the two emission points [6] and can be neglected in the present setup.

The tunneling limit $\Gamma \ll 1$ makes it relevant to change the perspective from a quasiparticle picture to an allelectron picture. An Andreev reflection, occuring with a small probability $\Gamma^{2}$, can be considered as a perturbation with respect to the ground state $|\overline{0}\rangle$ in the normal reservoirs (a filled Fermi sea of electrons at energies $E<-e V$ ). It creates an excitation consisting of a pair of electrons (see Fig. 21). Formally, performing a Bogoliubov transformation (i.e. $\gamma_{j \eta}(E)=c_{j \eta}^{\dagger}(-E)$, with $c_{j \eta}^{\dagger}(E)$ being a standard electron creation operator), the state of the quasiparticles going out from the superconductor becomes 20] to first order in $\Gamma$

$$
\left|\Psi_{\text {out }}\right\rangle=|\overline{0}\rangle+|\tilde{\Psi}\rangle+|\Psi\rangle
$$

where the states

$$
\begin{gathered}
|\tilde{\Psi}\rangle=\frac{i \Gamma}{4} \int_{0}^{e V} d E\left[\sum_{j=1,2} \sum_{\eta=A, B} c_{j \eta}^{\dagger}(E) c_{j \eta}(-E)\right]|\overline{0}\rangle \\
|\Psi\rangle=\frac{i \Gamma}{4} \int_{-e V}^{e V} d E\left[c_{1 A}^{\dagger}(E) c_{1 B}^{\dagger}(-E)\right. \\
\left.\quad+c_{2 A}^{\dagger}(E) c_{2 B}^{\dagger}(-E)\right]|\overline{0}\rangle
\end{gathered}
$$

describe orbitally entangled electron "wave packet" pairs, i.e. a superposition of pairs at different energies [21]. In a first quantized notation, the state $|\Psi\rangle$ is just the state in Eq. (2). We emphasize that the change from a quasiparticle to an all-electron picture, providing a clear picture of the entanglement, does not alter the physics.

The detection of the entanglement is done via the zero frequency current cross-correlators $S_{\alpha \beta}$ in Eq. (6). To obtain $S_{\alpha \beta}$, we insert the second quantization expression for the current operator [19], $\hat{I}_{\alpha \eta}(t)=$ $(e / h) \int d E d E^{\prime} \exp \left[i\left(E-E^{\prime}\right) t / \hbar\right] c_{\alpha \eta}^{\dagger}\left(E^{\prime}\right) c_{\alpha \eta}(E)$, into Eq. (6) and average with respect to the state $|\Psi\rangle$ in Eqs. (8) and (9). The beam splitters are taken into account by relating the $c_{j \eta^{-}}$operators in arms $1 A$ and $2 A$ $(1 B$ and $2 B)$ to the $c_{\alpha \eta^{-}}$-operators in the reservoirs $+A$ and $-A(+B$ and $-B)$ via the scattering matrix in Eq. (11). The average current, equal in all arms $\alpha \eta$, is $\left\langle I_{\alpha \eta}\right\rangle \equiv I=\left(e^{2} / 2 h\right) \Gamma^{2} V$, independent of the beam splitter transparency.

The ground state $|\overline{0}\rangle$ in Eq. (8) does not contribute to the correlator. Moreover, calculating the current correlator we find that the state $|\tilde{\Psi}\rangle$, describing two electrons
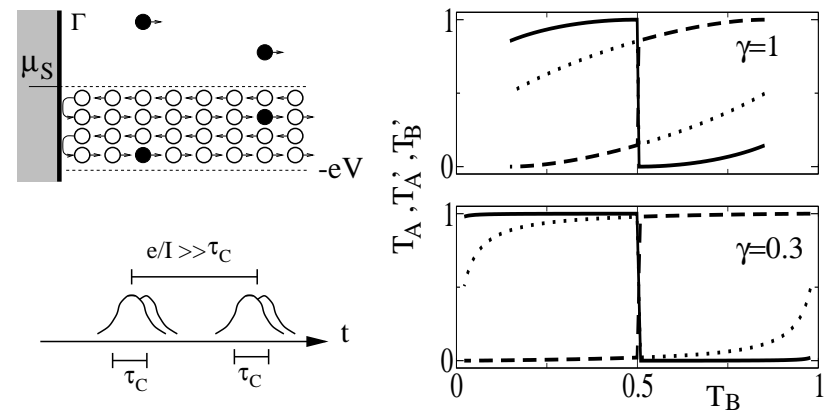

FIG. 2: Left, upper: In the filled stream of incoming holes (empty circles) from the normal reservoir, occasionally a hole is back-reflected as an electron (solid circles). The "missing hole" (i.e an electron) and the Andreev reflected electron constitute the pair emitted from the superconductor. Left, lower: The correlation time $\tau_{c}=\hbar / \mathrm{eV}$ (width of the wave packet) and the average time between emission of two subsequent pairs $e / I=\hbar / e V \Gamma^{2} \gg \tau_{c}$ of the current correlator. The small time difference $\hbar / \Delta \ll \tau_{c}$ between the emissions of the two electrons in the pair is shown as a split of the wavepacket. Right: The transmission probabilities $T_{A}$ (dashed), $T_{A}^{\prime}$ (dotted) and $T_{B}^{\prime}$ (solid) as a function of $T_{B}\left[T_{\eta}=\cos ^{2}\left(\phi_{\eta}\right)\right]$, giving optimal violation of the Bell inequalities for dephasing parameters $\gamma=1$ and $\gamma=0.3$.

emitted into the same normal lead, only contributes 13. to the cross correlator at order $\Gamma^{4}$ and can thus be neglected. From Eq. (6) we then find that $S_{\alpha \beta}\left(\phi_{A}, \phi_{B}\right)=$ $P_{0} P_{\alpha \beta}\left(\phi_{A}, \phi_{B}\right)$, where $P_{\alpha \beta}$ is given in Eq. (5), just as announced. We note that it is the structure of $|\Psi\rangle$ in 12 space, i.e. $\left|\Psi_{12}\right\rangle$, that determines the angle dependence of $P_{\alpha \beta}$, all properties of $\left|\Psi_{A B}\right\rangle$ just gives rise to the constant $P_{0}$. Moreover, the calculations show that the time dependent correlator $\left\langle\delta \hat{I}_{1 \alpha}(t) \delta \hat{I}_{2 \beta}(0)\right\rangle$ vanishes as $\left(\tau_{c} / t\right)^{2}$ for $t \gg \tau_{c}$. As is pointed out above, $S_{\alpha \beta}\left(\phi_{A}, \phi_{B}\right)$ can thus be inserted in Eq. (44) and be used to violate the BI in Eq. (3).

Until now, ideal conditions have been considered. One possible source of disturbances is dephasing. In our system, dephasing can quite generally be expressed in terms of a density matrix $\rho=[|11\rangle\langle 11|+| 22\rangle\langle 22|+\gamma(|11\rangle\langle 22|+$ $|11\rangle\langle 22|)] / 2$, where the off-diagonal elements, giving rise to the entanglement, are suppressed by a phenomenological dephasing parameter $0 \leq \gamma \leq 1$. The correlators $E\left(\phi_{A}, \phi_{B}\right)$ in Eq. (4) then take the form

$$
E\left(\phi_{A}, \phi_{B}\right)=\cos \left(2 \phi_{A}\right) \cos \left(2 \phi_{B}\right)+\gamma \sin \left(2 \phi_{A}\right) \sin \left(2 \phi_{B}\right) \text {. }
$$

By adjusting the four angles $\phi_{A}, \phi_{A}^{\prime}, \phi_{B}$ and $\phi_{B}^{\prime}$ we find the maximal Bell parameter in Eq. (3) is

$$
S=2 \sqrt{1+\gamma^{2}}
$$

which violates the BI for any $\gamma>0$. The optimal violation angles, all in the first quadrant, $\operatorname{are} \tan \left(2 \phi_{A}\right)=$ $-\gamma \cot \left(\phi_{S}\right), \tan \left(2 \phi_{A}^{\prime}\right)=\gamma \tan \left(\phi_{S}\right)$ and $\tan \left(\phi_{B}-\right.$ $\left.\phi_{B}^{\prime}\right)=\operatorname{sign}\left[\cos \left(2 \phi_{A}\right)\right]\left[\left(\tan ^{2}\left(\phi_{S}\right)+\gamma^{2}\right) /\left(\gamma^{2} \tan ^{2}\left(\phi_{S}\right)+\right.\right.$ 
1) $]^{1 / 2}$, where $\phi_{S}=\phi_{B}+\phi_{B}^{\prime}$ can be chosen at will. The corresponding transmission probabilities $T_{\eta}=\cos ^{2}\left(\phi_{\eta}\right)$ are shown for $\gamma=1$ and $\gamma=0.3$ in Fig. 2

The BI can thus in principle be violated for any amount of dephasing. However it might be difficult to produce beam splitters which can reach all transmission probabilities between 0 and 1 . This is not a serious problem in the absence of dephasing, $\gamma=1$, a violation can be obtained for a large, order of unity, fraction of the "transmission probability space". However, in the limit of strong dephasing, $\gamma \ll 1$, the set of transmission probabilities for optimal violation contain transmissions close to both 0 and 1, see Fig. 2 Expecting unity transmission to be most complicated to reach experimentally, we note that by instead choosing transmission probabilities $T_{A}=T_{B}=0, T_{B}^{\prime}=1 / 2$ and $T_{A}^{\prime} \ll \gamma$, the inequality in Eq. (3) becomes $2\left|1+\gamma T_{A}^{\prime}\right| \leq 2$. This gives a violation, although not maximal, for all $\gamma \ll 1$.

Apart from dephasing there are several other effects such as additional scattering phases, impurity scattering or asymmetric tunnel barriers, which might alter the possibility to violate the BI. It turns out that all these effects can be taken into account by replacing $\gamma \rightarrow \gamma^{\prime} \cos \left(\phi_{0}\right)$ in Eq. (11), with the important conclusion that none of these effects will destroy the possibility to violate the BI.

The phase factor $\phi_{0}$ is the sum of possible scattering phases from the beam splitters [the scattering amplitudes in Eq. (1) are taken real], phases $\sim k_{F} \Delta L$ due to a difference in length, $\Delta L$, between the normal arms (see Fig. 10), scattering phases from weak impurities and a possible phase difference between the superconductor at the two tunnel contacts 1 and 2 . As a consequence e.g. the superconducting phase (in a loop geometry) can be modulated to compensate for the other phases.

The factor $\gamma^{\prime}$ plays the same role as dephasing. One possible contribution to $\gamma^{\prime}$ is energy dependent phases which oscillate rapidly on a scale of $e V$, suppressing the entangled part of the current correlator. For different lengths of the normal arms, there is always a phase $\sim E \Delta L / \hbar v_{F}$. This phase can however be neglected for $\Delta L \ll \hbar v_{F} / e V$, which for $e V \ll \Delta$ is fulfilled for $\Delta L$ smaller than the superconducting coherence length $\hbar v_{F} / \Delta$. Another possibility is that, due to asymmetries of the tunnel barriers $\Gamma_{1} \neq \Gamma_{2}$, the amplitude for the process where the pair is emitted to $|11\rangle$ is different from the process where it is emitted to $|22\rangle$. This gives rise to a state, in 12-space, $\left.\left(\Gamma_{1}|11\rangle+\Gamma_{2}|22\rangle\right) / \sqrt{\Gamma_{1}^{2}+\Gamma_{2}^{2}}\right)$. In this case 22] $\gamma^{\prime}=2 \Gamma_{1} \Gamma_{2} /\left(\Gamma_{1}^{2}+\Gamma_{2}^{2}\right)$. Thus, it is in principle possible to violate $\mathrm{BI}$ for arbitrary asymmetry.

We finally point out that the constraint on single mode beam splitter can not easily be relaxed. A multimode beam splitter will, in the suggested setup, probably have a different transparency dependence for the different modes. This decreases the space of accessible angles and will eventually make the violation of the BI impossible.

In conclusion, we have investigated a spin-independent scheme to generate and detect two-particle orbital entanglement in a mesoscopic normal-superconductor system. The cross-correlator between the currents in the two leads depends in a nonlocal way on transparencies of beam splitters in the two leads. These nonlocal correlations arises due to the entanglement of the injected pair. For appropriate choices of transparencies, the correlators give a violation of a $\mathrm{BI}$ for arbitrary strong dephasing.

We acknowledge discussions with Fabio Taddei and Valerio Scarani. This work was supported by the Swiss National Science Foundation and the program for Materials with Novel Electronic Properties.

[1] A. Einstein, B. Podolsky and N. Rosen, Phys. Rev. 47, 777 (1935); D. Bohm and Y. Aharonov, Phys. Rev. 108, 1070 (1957).

[2] J.S. Bell, Physics 1, 195 (1964); Rev. Mod. Phys. 38, 447 (1966).

[3] For recent experiments, see e.g. W. Tittel et al., Phys. Rev. Lett 81, 3563 (1998); G. Weihs et al., ibid 81 5039, (1998).

[4] N. Gisin et al., Rev. Mod. Phys. 74, 145 (2002).

[5] A. Steane, Rep. Prog. Phys. 61, 117 (1998).

[6] P. Recher, E. V. Sukhorukov, and D. Loss, Phys. Rev. B 63, 165314 (2001).

[7] P. Recher and D. Loss, Phys. Rev. B 65165327 (2002); C. Bena et al, Phys. Rev. Lett 89, 037901 (2002).

[8] G. Burkard, D. Loss and E.V. Sukhorukov, Phys. Rev. B 61, 16303 (2000); F. Taddei and R. Fazio, ibid 65, 134522 (2002); J. C. Egues, G. Burkard, and D. Loss, Phys. Rev. Lett. 89, 176401 (2002).

[9] G.B. Lesovik, T. Martin and G. Blatter, Eur. Phys. J. B 24, 287 (2001).

[10] N.M. Chtchelkatchev et al., Phys. Rev. B 66, 161320 (2002).

[11] The two parts of the superconductor can be contacted e.g. via a loop.

[12] R. Liu et al., Nature 391, 263 (1998); W.D. Oliver et al., Science 284, 299 (1999).

[13] P. Samuelsson and M. Büttiker, Phys. Rev. Lett. 89, 046601 (2002); Phys. Rev. B 66, 201306 (2002).

[14] J.F. Clauser et al., Phys. Rev. Lett. 23, 880 (1969).

[15] A. Aspect, P. Grangier, and G. Roger, Phys. Rev. Lett. 49, 91 (1982).

[16] In contrast to Ref. [10] there is no constraint on the measurement time in our scheme.

[17] S. Datta, P.F. Bagwell, and M.P. Anantram, Phys. LowDim. Struct. 3, 1 (1996); C.W.J. Beenakker, Rev. Mod. Phys, 69, 731 (1997).

[18] The same result can be obtained using the tunneling Hamiltonian formalism in ref. [6].

[19] M. Büttiker, Phys. Rev. B. 46, 12485 (1992); Ya. M. Blanter, and M. Büttiker, Phys. Rep. 336, 1 (2000).

[20] For a related approach in optics, see e.g. Z.Y Ou et al., Phys. Rev. A. 41, 566 (1990).

[21] In contrast to Refs. [8], in our setup there is no additional constraint on the energy spectrum of the entangled electrons.

[22] N. Gisin, Phys. Lett. A 154, 201 (1991); A. Abouraddy et. al., Phys. Rev. A 64, 050101 (2001). 